\title{
SOME VALUES FOR CONSTANT-SUM AND BILATERAL COOPERATIVE GAMES
}

Abstract. We prove new axiomatizations of the Shapley value and the Banzhaf value, defined on the class of nonnegative constant-sum games with nonzero worth of the grand coalition as well as on nonnegative bilateral games with nonzero worth of the grand coalition. A characteristic feature of the latter class of cooperative games is that for such a game any coalition and its complement in the set of all players have the same worth. The axiomatizations are then generalized to the entire class of constant-sum or bilateral games, respectively. Moreover, a new axiomatization of the Deegan-Packel value on the set of all cooperative games is presented and possibilities of creation of its version in those special cases are discussed.

Introduction. One of the most important problems in game theory is axiomatization of the main, well-known solutions (values) of cooperative games. The famous theorems formulated and proved by L. S. Shapley (1953), J. Deegan and E. W. Packel (1978), H. P. Young (1985), E. Lehrer (1988), J. A. M. Potters (1991) and L. M. Ruiz et al. (1996) belong to the fundamental results in this area. Besides the general approach applied in the above-mentioned theorems, in some papers also other forms of axiomatization of those values for some specific types of cooperative games are discussed. G. Owen (1977) gave an axiomatization of the Shapley value for a game with a priori unions (this research for other values was continued among others by A. Młodak (2003)). S. C. Littlechild and G. Owen (1976) as well as later M. Vázquez-Brage et al. (1997) investigated some properties of the Shapley value for the so-called "airport" games, which is a class of allocation cost games.

2000 Mathematics Subject Classification: Primary 91A12.

Key words and phrases: cooperative game, constant-sum game, bilateral game, value, Banzhaf value, Deegan-Packel value. 
The present paper contains an extension of the analysis conducted by A. B. Khmelnitskaya (2003) and concerning constant-sum cooperative games. She has noted that H. P. Young's (1985) axiomatization for the Shapley value, which characterizes it by the efficiency, symmetry and marginalism axioms, is still valid for the Shapley value defined on the subclass of nonnegative constant-sum games with nonzero worth of the grand coalition (i.e. one containing all the players of the game) as well as on the entire class of constant-sum games. We give some parallel theorems for another cooperative game value, namely the Banzhaf value restricted to the constant-sum games (also in its nonnegative version). Its axiomatization consists of the marginalism, symmetry and amalgamation properties.

Next we analyze another type of cooperative game called a bilateral game. The main feature of this model is the coincidence of the worth of any coalition of such a game with the worth of its complement in the grand set of players $N$. If the worth of each coalition is nonnegative (and nonzero for the grand coalition), then the game is called a nonnegative bilateral game. We give an axiomatization of the above-mentioned value (and also of the Shapley value) defined on this class of games which is quite similar to the one in the case of constant-sum games (and in the case of its nonnegative variant). It is worth noting that bilateral games also have an important practical interpretation. The fact that any coalition and its complement in the grand coalition receive the same benefit implies that individual participants are relatively important. As a consequence of one participant's transfer from one coalition to another all other players can lose or all can gain.

Finally, we consider the Deegan-Packel value. We prove it is a unique value satisfying the quasi-efficiency, zero-player, quasi-marginalism and symmetry axioms. The concept of quasi-marginalism is in some sense similar to the notion of "classical" marginalism. Instead of marginal utility, we assume the dependence of a value for a given player only on the worth of all the coalitions he belongs to. Finally, we discuss the possibility of constructing an axiomatization of this value defined on the set of constant-sum and bilateral games.

1. Preliminaries. First we recall the fundamental definitions and facts connected with cooperative game theory and specific models which will be considered in this research.

Let $n \geq 2$ be a fixed natural number. An $n$-person transferable utility cooperative game (briefly, a TU-game) is defined by the set of players $N=$ $\{1, \ldots, n\}$ (the grand coalition) and by a function $v: 2^{N} \rightarrow \mathbb{R}$, with $v(\emptyset)=0$, called the characteristic function of the game. Therefore, when $N$ is fixed, a TU-game $(N, v)$ can be identified with $v$. The cardinality of a coalition $S \subseteq N$ will be denoted by $|S|$. 
Let $G_{N}$ be the set of all $n$-person games $v$. The sum of $v, w \in G_{N}$ is defined by $(v+w)(S)=v(S)+w(S)$ for all $S \subseteq N$. Similarly, if $v \in G_{N}$ and $a$ is a nonzero real number then we define $(a v)(S)=a \cdot v(S)$ for all $S \subseteq N$. If $v(S \cup\{i\})=v(S \cup\{j\})$ for all $S \subseteq N \backslash\{i, j\}$ and any $i, j \in N, i \neq j$, then the game $v$ is said to be symmetric. The dual game to $v$ is defined by $v^{*}(S)=v(N)-v(N \backslash S)$ for all $S \subseteq N$.

Fix any $T \subseteq N$. The unanimity game $u_{T} \in G_{N}$ is given by $u_{T}(S)=1$ if $T \subseteq S$ and $u_{T}(S)=0$ otherwise, for every $S \subseteq N$. The basic game $w_{T} \in G_{N}$ is defined by $w_{T}(S)=1$ if $S=T$ and $w_{T}(S)=0$ otherwise, for every $S \subseteq N$.

Definition 1. A game $v \in G_{N}$ is called a constant-sum game if for every $S \subseteq N$,

$$
v(S)+v(N \backslash S)=v(N) .
$$

Thus any constant-sum game is self-dual, i.e. $v=v^{*}$. The set of all constant-sum games will be denoted by $\Gamma_{N}$. One can see that the transfer of a player from $S$ to $N \backslash S$ does not change the general balance of the game.

Definition 2. A game $v \in G_{N}$ is called a nonnegative constant-sum game if $v \in \Gamma_{N}$ and $v(N) \neq 0$ and $v(S) \geq 0$ for every $S \subseteq N$.

Thus, we assume that the worth of every coalition is nonnegative and nonzero in the "grand" case. The set of all nonnegative constant-sum games will be denoted by $\Gamma_{N}^{+}$.

Now we introduce two new (but in some sense similar) types of games.

Definition 3. A game $v \in G_{N}$ is called a bilateral game if for every $S \subseteq N, S \neq \emptyset, N$,

$$
v(S)=v(N \backslash S) .
$$

The set of all bilateral games will be denoted by $\Im_{N}$. So, the worth of a given coalition is equal to the worth of its complement in $N$. This increases the importance of an individual player. His decision regarding a prospective change of coalition influences the benefits of all the other participants.

Definition 4. A game $v \in G_{N}$ is called a nonnegative bilateral game if $v \in \Im_{N}$ and $v(N) \neq 0$ and $v(S) \geq 0$ for every $S \subseteq N$.

The set of all nonnegative bilateral games will be denoted by $\Im_{N}^{+}$.

A value of the game $v \in G_{N}$ is defined to be a function $\varphi(v)=\left(\varphi_{1}(v), \ldots\right.$, $\left.\varphi_{n}(v)\right)$ which assigns to the game $v$ a vector from $\mathbb{R}^{n}$. An $n$-dimensional vector $x=\left(x_{1}, \ldots, x_{n}\right) \in \mathbb{R}^{n}$ is called a preimputation if

$$
\sum_{i=1}^{n} x_{i}=v(N) .
$$

If, additionally, $x_{i} \geq v(\{i\})$ for all $i \in N$, then $x$ is called an imputation. 
It seems useful to formulate here several fundamental properties of values of cooperative games, which will be used to construct some axiomatizations, restricted to special types of games.

Let $\varphi$ be a value on $G_{N}$ and $v \in G_{N}$ be any $n$-person game.

EFFICIENCY AXIOM. A value $\varphi$ is efficient if for any game $v$,

$$
\sum_{i=1}^{n} \varphi_{i}(v)=v(N)
$$

This means that the vector $\varphi(v)$ is a preimputation.

QUASI-EFFICIENCY AXIOM. A value $\varphi$ is quasi-efficient if for any game $v$,

$$
\sum_{i=1}^{n} \varphi_{i}(v)=\sum_{S \subseteq N} v(S) .
$$

ZERO-PLAYER AXIOM. If $i$ is the zero-player of a game $v$ (i.e. if $v(S)=0$ for any $S \subseteq N$ with $i \in S)$, then $\varphi_{i}(v)=0$.

Let $\sigma: N \rightarrow N$ be any permutation of the set of players and $\sigma v \in G_{N}$ be the game $\sigma v(S)=v(\sigma(S))$, where $\sigma(S)=\{\sigma(i): i \in S\}$ for any $S \subseteq N$.

Symmetry AXIOM. A value $\varphi$ is said to be symmetric if for any game $v$, any $\sigma$ and any $i \in N, \varphi_{\sigma(i)}(\sigma v)=\varphi_{i}(v)$.

To formulate the next axiom, we recall the definition of amalgamation of two players introduced by E. Lehrer (1988).

Definition 5. Amalgamation of any two different players $i, j \in N$ of an $n$-person game $v$ is a transformation of the game $v$ into an $(n-1)$-person game $v_{(i j)}$ with the set of players $(N \backslash\{i, j\}) \cup\{p\}$, where $p$ denotes a player represented by the coalition $\{i, j\}$. The characteristic function of the latter game is defined by

$$
v_{(i j)}(S)= \begin{cases}v(S) & \text { if } p \notin S, \\ v((S \backslash\{p\}) \cup\{i, j\}) & \text { if } p \in S,\end{cases}
$$

for any set $S \subseteq(N \backslash\{i, j\}) \cup\{p\}$.

For a better description of further notions, it is also necessary to define the set of all games with grand coalition being a subset of $N$, that is,

$$
\widetilde{G}_{N}=\bigcup_{T \subseteq N} G_{T}
$$

Analogously, one can define the sets $\widetilde{\Gamma}_{N}, \widetilde{\Gamma}_{N}^{+}, \widetilde{\Im}_{N}, \widetilde{\Im}_{N}^{+}$. Of course, in the same way as for $G_{N}$, we can define a value $\varphi$ of a game belonging to any of the above-mentioned classes.

Amalgamation Axiom. For any $i, j \in N, i \neq j$, and $v \in \widetilde{G}_{N}, \varphi_{p}\left(v_{(i j)}\right)$ $=\varphi_{i}(v)+\varphi_{j}(v)$. 
Marginalism AXIOM. A value $\varphi$ is said to be marginalist if for all $v \in$ $G_{N}$ and every $i \in N, \varphi_{i}(v)$ depends only upon the $i$ th marginal utility vector, that is,

$$
\varphi_{i}(v)=f_{i}(v(S \cup\{i\})-v(S))_{S \subseteq N \backslash\{i\}},
$$

where $f_{i}$ is a function of $2^{n-1}$ variables, i.e. $f_{i}: \mathbb{R}^{2^{n-1}} \rightarrow \mathbb{R}$.

QUASI-MARGINALISM AXIOM. A value $\varphi$ is said to be quasi-marginalist if for all $v \in G_{N}$ and every $i \in N, \varphi_{i}(v)$ depends only on the values of the characteristic function of this game for coalitions containing player $i$ (or, in other words, on the $i$ th quasi-marginal utility vector), that is,

$$
\varphi_{i}(v)=f_{i}(v(S \cup\{i\}))_{S \subseteq N \backslash\{i\}},
$$

where $f_{i}$ is a function of $2^{n-1}$ variables, i.e. $f_{i}: \mathbb{R}^{2^{n-1}} \rightarrow \mathbb{R}$.

We recall three types of well-known values of cooperative games which are the main object of our interest.

Definition 6 (L. S. Shapley (1953)). The Shapley value of player $i \in N$ in a game $v \in G_{N}$ is defined as

$$
\operatorname{Sh}_{i}(v)=\sum_{S \subseteq N \backslash\{i\}} \frac{|S| !(n-|S|-1) !}{n !}(v(S \cup\{i\})-v(S)) .
$$

The value $\operatorname{Sh}(v)$ is efficient and in some cases it is also an imputation.

Definition 7 (J. F. Banzhaf III (1965)). The Banzhaf value of player $i \in N$ in a game $v \in G_{N}$ is defined as

$$
B_{i}(v)=\frac{1}{2^{n-1}} \sum_{S \subseteq N \backslash\{i\}}(v(S \cup\{i\})-v(S)) .
$$

Definition 8 (J. Deegan and E. W. Packel (1978)). The Deegan-Packel value of player $i \in N$ in a game $v \in G_{N}$ is defined as

$$
\operatorname{DP}_{i}(v)=\sum_{S \subseteq N, i \in S} \frac{v(S)}{|S|} .
$$

The value $\operatorname{DP}(v)$ is not efficient, but it is quasi-efficient.

The starting point of our considerations is the following theorem formulated and proved by A. B. Khmelnitskaya (2003).

THEOREM 1. The only efficient, symmetric and marginalist value defined on $\widetilde{\Gamma}_{N}^{+}$is the Shapley value.

In the above-cited paper this main idea was easily generalized to the set $\Gamma_{N}$. The three axioms mentioned are sufficient and necessary to uniquely describe the Shapley value also in this case. It is worth noting that Theorem 1 
is no longer valid for $G_{N}$. As a counterexample one can consider the threeperson game $N=\{1,2,3\}$ with characteristic function $v(\{1\})=v(\{2\})=$ $v(\{3\})=v(\{2,3\})=0$ and $v(\{1,2\})=v(\{1,3\})=v(\{1,2,3\})=1$. Then we have $\operatorname{Sh}(v)=(2 / 3,1 / 6,1 / 6)$. On the other hand, the normalized Banzhaf values are given as $B^{*}(v)=\left(B_{1}(v) / \beta, B_{2}(v) / \beta, B_{3}(v) / \beta\right)=(3 / 5,1 / 5,1 / 5)$, where $\beta=B_{1}(v)+B_{2}(v)+B_{3}(v)=5 / 4$. Both values, $\operatorname{Sh}(v)$ and $B^{*}(v)$, are efficient, symmetric and marginalist, but have quite different values. This contradicts the uniqueness of the Shapley value in this case.

2. Main results. As stated earlier, the concept of a value may refer to games representing various specific classes also with different grand coalitions (cf. formula (1) and the remarks following it, for example). This remark will be used in our approach.

To obtain an effective axiomatization of the Banzhaf value defined on the set $\Gamma_{N}^{+}$it is sufficient to replace the efficiency axiom (which is generally not satisfied by this value) with the amalgamation property. Thus we obtain the following result.

THEOREM 2. The only value defined on $\widetilde{\Gamma}_{N}^{+}$and satisfying the amalgamation, symmetry and marginalism axioms is the Banzhaf value.

Theorem 2 is also valid in the case of the set $\widetilde{\Gamma}_{N}$. Analogous statements on $\Im_{N}^{+}$and $\widetilde{\Im}_{N}^{+}$(and also on $\Im_{N}$ and $\widetilde{\Im}_{N}$, respectively) are presented in Theorems 3 and 4 .

THEOREM 3. A value defined on $\Im_{N}^{+}$satisfies the efficiency, symmetry and marginalism axioms if and only if it coincides with the Shapley value.

THEOREM 4. The only value defined on $\widetilde{\Im}_{N}^{+}$and satisfying the amalgamation, symmetry and marginalism axioms is the Banzhaf value.

Because the Deegan-Packel value is not marginalist, there seems to be no simple axiomatization of this value defined on the above-mentioned class of games. But in this case a more general theorem holds which uses quasiefficiency and quasi-marginalism instead of the classical efficiency and marginalism axioms.

Theorem 5. The Deegan-Packel value is a unique value defined on $G_{N}$ and satisfying the quasi-efficiency, zero-player, symmetry and quasimarginalism axioms.

At the end of this paper, on the basis of the proof of this theorem, we discuss in detail some problems concerning axiomatization of this value on $\Gamma_{N}$ and $\Im_{N}$ (and consequently, on $\Gamma_{N}^{+}$and $\Im_{N}^{+}$). We also propose a partial solution involving only the quasi-efficiency and quasi-marginalism axioms satisfied by a value on $\Gamma_{N}$. 
3. Proofs. We begin with the proofs of Theorems 2 and 4 . Since the methods used are very similar, the two theorems will be proved simultaneously.

3.1. Proof of Theorems 2 and 4. $(\Leftarrow)$ It is well-known (cf. E. Lehrer (1988)) that the Banzhaf value satisfies the three axioms.

$(\Rightarrow)$ We know (L. S. Shapley (1953)) that any game $v \in G_{N}$ can be uniquely represented as a linear combination of unanimity games, that is,

$$
v=\sum_{\emptyset \neq T \subseteq N} \lambda_{T} u_{T}
$$

for some real constants $\lambda_{T}, T \subseteq N$. Note that for any $v, v^{\prime} \in G_{N}$ and any real number $\alpha$ we have $\left(v+v^{\prime}\right)^{*}=v^{*}+v^{*}$ and $(\alpha v)^{*}=\alpha \cdot v^{*}$. Therefore the game dual to $v$ is represented via the dual unanimity basis, i.e.

$$
v^{*}=\sum_{\emptyset \neq T \subseteq N} \lambda_{T} u_{T}^{*}
$$

Let $v_{c} \in \Gamma_{N}^{+}$be any nonnegative constant-sum game. Since clearly $v_{c}=$ $\left(v_{c}+v_{c}^{*}\right) / 2$, formulas $(2)$ and (3) directly imply that the game $v_{c}$ can be represented in the form

$$
v_{c}=\sum_{\emptyset \neq T \subseteq N} \lambda_{T}^{(c)} u_{T}^{(c)}
$$

with some reals $\lambda_{T}^{(c)}$, where for every $S \subseteq N$,

$$
u_{T}^{(c)}(S)=\frac{u_{T}(S)+u_{T}^{*}(S)}{2}= \begin{cases}1, & T \subseteq S, \\ 1 / 2, & T \cap S \neq \emptyset, T \not \subset S, \\ 0, & T \cap S=\emptyset .\end{cases}
$$

Let $v_{b} \in \Im_{N}^{+}$be any nonnegative bilateral game. Then, after putting $v=v_{b}$ in (2) and considering the values of both sides of (2) for arbitrary coalitions $S$ and $N \backslash S$ with $S \neq \emptyset$ and $S \neq N$ we easily see that

$$
v_{b}=\sum_{\emptyset \neq T \subseteq N} \lambda_{T}^{(b)} u_{T}^{(b)}
$$

with some reals $\lambda_{T}^{(b)}$, where $u_{T}^{(b)}(\emptyset)=0, u_{T}^{(b)}(N)=1 / 2$ and for every $S \subseteq N$, $S \neq \emptyset$ and $S \neq N$,

$$
u_{T}^{(b)}(S)=\frac{u_{T}(S)+u_{T}(N \backslash S)}{2}= \begin{cases}1 / 2, & T \subseteq S \text { or } T \subseteq N \backslash S, \\ 0 & \text { otherwise. }\end{cases}
$$

Thus, every player $i \notin T$ is a dummy-player in both games $u_{T}^{(c)}$ and $u_{T}^{(b)}$, that is, $u_{T}^{(x)}(S \cup\{i\})-u_{T}^{(x)}(S)=0$ for every $S \subseteq N \backslash\{i\}$ and $T \subseteq N \backslash\{i\}$, $x=c, b$. It is easy to check that for every $i \in N$ the Banzhaf values of games 
derived by transformation of unanimity games are

$$
B_{i}\left(u_{T}\right)=B_{i}\left(u_{T}^{*}\right)=B_{i}\left(u_{T}^{(c)}\right)= \begin{cases}1 / 2^{|T|-1}, & i \in T, \\ 0, & i \notin T .\end{cases}
$$

and

$$
\begin{aligned}
B_{i}\left(u_{T}^{(b)}\right)= & \frac{1}{2^{n-1}} u_{T}^{(b)}(\{i\})+\frac{1}{2^{n-1}}\left(u_{T}^{(b)}(N)-\frac{u_{T}(N \backslash\{i\})+u_{T}(\{i\})}{2}\right) \\
& +\frac{1}{2^{n}} \sum_{\substack{S \subseteq N \backslash\{i\} \\
S \neq \emptyset, N \backslash\{i\}}}\left(u_{T}(S \cup\{i\})+u_{T}(N \backslash(S \cup\{i\}))\right. \\
& \left.-u_{T}(S)-u_{T}(N \backslash S)\right) \\
= & \begin{cases}1 / 2^{n}, & i \in T, \\
0, & i \notin T .\end{cases}
\end{aligned}
$$

Because the coefficients $\lambda_{T}$ in the general formulas (4) and (5) are not necessarily all nonnegative, we cannot apply the induction procedure immediately (reduction of the number of coefficients in (4) and (5) may lead outside the classes $\Gamma_{N}^{+}$and $\Im_{N}^{+}$respectively). Applying an idea of A. B. Khmelnitskaya (2003) we take the following approach. For each $t=1, \ldots, n$ define (6) $\quad \lambda_{t}^{(x)}=\max \left\{\max _{T \subseteq N:|T|=t} \lambda_{T}^{(x)}, 0\right\} \quad$ and $\quad \eta_{T}^{(x)}=\lambda_{t}^{(x)}-\lambda_{T}^{(x)} \geq 0, \quad T \subseteq N$.

Consider two symmetric games

$$
\mu_{x}=\sum_{t=1}^{n} \lambda_{t}^{(x)} \sum_{\emptyset \neq T \subseteq N,|T|=t} u_{T}^{(x)},
$$

$x=c, b$. One can easily check that each $u_{T}^{(c)}$ is a constant-sum game and each $u_{T}^{(b)}$ is a bilateral game. Moreover, all $\lambda_{t}^{(x)} \geq 0$ because of (6). Therefore, by $(2), \mu_{c} \in \Gamma_{N}^{+}$and $\mu_{b} \in \Im_{N}^{+}$. Hence, taking into account (4)-(7) for $x=c, b$ we get the equalities

$$
v_{x}=\mu_{x}-\sum_{\emptyset \neq T \subseteq N} \eta_{T}^{(x)} u_{T}^{(x)}
$$

with all $\eta_{T}^{(x)} \geq 0$. Therefore, in both cases $x=c$ and $x=b$, every summand in (8) belongs to $\Gamma_{N}^{+}$and $\Im_{N}^{+}$, respectively.

Let $q_{c}$ and $q_{b}$ denote the minimum numbers of summands in (8) for $x=c$ and $x=b$ respectively. Thus

$$
v_{x}=\mu_{x}-\sum_{k=1}^{q_{x}} \eta_{T_{k}}^{(x)} u_{T_{k}}^{(x)}
$$

for $x=c, b$, where all $\eta_{T_{k}}^{(x)}>0$. 
We will apply a double induction on the index $q_{x}(x=c, b)$ and the number $n$ of players. One can easily deduce that Theorems 2 and 4 hold for $n=2$. Assume that $n>2$. Let $\xi$ be a value defined on $\widetilde{\Gamma}_{N}^{+}$and $\widetilde{\Im}_{N}^{+}$ satisfying the amalgamation, symmetry and marginalism axioms. If $q_{x}=0$ then $v_{x}=\mu_{x}, x=c, b$. Both $\mu_{c}$ and $\mu_{b}$ are symmetric games, therefore by the symmetry axiom, for any $i, j \in N, i \neq j$, we have $\xi_{i}\left(v_{x}\right)=\xi_{j}\left(v_{x}\right)$. Amalgamate players $i$ and $j$. Then using the fact that $v_{(i j) x}$ is an $(n-1)$ person game and the induction hypothesis with respect to $n$ we conclude that

$2 \xi_{i}\left(v_{x}\right)=\xi_{i}\left(v_{x}\right)+\xi_{j}\left(v_{x}\right)=\xi_{p}\left(v_{(i j) x}\right)=B_{p}\left(v_{(i j) x}\right)=B_{i}\left(v_{x}\right)+B_{j}\left(v_{x}\right)=2 B_{i}\left(v_{x}\right)$.

Thus we have proved that $\xi_{i}\left(v_{x}\right)=B_{i}\left(v_{x}\right)$ for $x=c, b$ and $i \in N$ if $q_{x}=0$.

Now suppose that for $x=c, b, \xi\left(v_{x}\right)=B\left(v_{x}\right)$ for all games $v_{x}$ with indices not greater than some $q_{x} \geq 0$ (here $v_{c} \in \Gamma_{N}^{+}$and $v_{b} \in \Im_{N}^{+}$in the cases $x=c, b$ respectively). Consider next two arbitrary games $z_{c} \in \Gamma_{N}^{+}$and $z_{b} \in \Im_{N}^{+}$with indices $q_{c}+1$ and $q_{b}+1$, respectively. For all $h, i \in \Theta=$ $\bigcap_{k=1}^{q_{x}+1} T_{k}$ the symmetry axiom implies that $\xi_{h}\left(z_{x}\right)=\xi_{i}\left(z_{x}\right)$. We will prove that $\xi_{j}\left(z_{x}\right)=B_{j}\left(z_{x}\right)$ for $j \notin \Theta, x=c, b$. Consider the game

$$
z_{x}^{\langle j\rangle}=\mu_{x}-\sum_{k: j \in T_{k}} \eta_{T_{k}}^{(x)} u_{T_{k}}^{(x)} .
$$

The index of $z_{x}^{\langle j\rangle}$ is at most $q_{x}$ and hence, by induction hypothesis with respect to $q_{x}$, we have $\xi\left(z_{x}^{\langle j\rangle}\right)=B\left(z_{x}^{\langle j\rangle}\right), x=c, b$. Since $j \notin \Theta$, player $j$ is a dummy-player in all games $u_{T}^{(x)}$ with $j \notin T$. Consequently, the $j$ th marginal utility vectors of the games $z_{x}$ and $z_{x}^{\langle j\rangle}$ coincide and hence, by marginalism for $\xi$ and for the Banzhaf value, for all $j \notin \Theta$ we have $\xi_{j}\left(z_{x}\right)=\xi_{j}\left(z_{x}^{\langle j\rangle}\right)=$ $B_{j}\left(z_{x}^{\langle j\rangle}\right)=B_{j}\left(z_{x}\right), x=c, b$.

Amalgamate now two players $i \in \Theta$ and $j \notin \Theta$. Then by induction hypothesis on $n$ and the amalgamation property of $\xi$ we have $\xi_{i}\left(z_{x}\right)+\xi_{j}\left(z_{x}\right)=$ $\xi_{p}\left(z_{(i j) x}\right)=B_{p}\left(z_{(i j) x}\right)$. Moreover, $\xi_{j}\left(z_{x}\right)=B_{j}\left(z_{x}\right)$ as shown before. Therefore $\xi_{i}\left(z_{x}\right)=B_{p}\left(z_{(i j) x}\right)-\xi_{j}\left(z_{x}\right)=B_{p}\left(z_{(i j) x}\right)-B_{j}\left(z_{x}\right)=B_{i}\left(z_{x}\right), x=c, b$.

Thus, the proof of Theorems 2 and 4 is complete.

These results can be easily generalized to the sets $\widetilde{\Gamma}_{N}$ and $\widetilde{\Im}_{N}$, respectively. To do this, it is sufficient to conduct the considerations in the same way but analyzing in the relevant places of the above proof the games on $\Gamma_{N}$ and $\Im_{N}$ as well as solutions on $\widetilde{\Gamma}_{N}$ and $\widetilde{\Gamma}_{N}^{+}$.

3.2. Proof of Theorem 3. This proof is quite similar to the one by A. Khmelnitskaya in the case of the Shapley value defined on $\Gamma_{N}^{+}$and uses some methods applied above. 
$(\Leftarrow)$ It is well-known (cf. L. S. Shapley (1953)) that the Shapley value satisfies the three stated conditions.

$(\Rightarrow)$ Let $v_{b} \in \Im_{N}^{+}$be any nonnegative bilateral game. Then, as shown in the proof of Theorem 4, it can be represented in the form (5). We recall our observation in that proof, that every player $i \notin T$ is a dummy-player in the game $u_{T}^{(b)}$ and if $|T| \geq 2$ then any players $i, j \in T, i \neq j$, are symmetric in this game. Therefore, the Shapley value of any player $i \in N$ in this case is

$$
\operatorname{Sh}_{i}\left(u_{T}^{(b)}\right)= \begin{cases}1 /(2|T|) & \text { if } i \in T, \\ 0 & \text { if } i \notin T .\end{cases}
$$

Define $\eta_{T}^{(b)}$ as in (6) and $\mu_{b}$ as in (7). Thus the game $v_{b}$ can be represented in the form (8) for $x=b$. Let $q_{b}$ be the number of nonzero coefficients under the summation sign in (8) for $x=b$. Thus

$$
v_{b}=\mu_{b}-\sum_{k=1}^{q_{b}} \eta_{T_{k}}^{(b)} u_{T_{k}}^{(b)}
$$

with all $\eta_{T_{k}}^{(b)} \neq 0$.

Let $\xi$ be an efficient, symmetric and marginalist value on $\Im_{N}^{+}$. Repeating the induction procedure with respect to $q_{b}$ applied in the proof of Theorem 4, we have $v_{b}=\mu_{b}$ for $q_{b}=0$ and because of symmetry of the latter game we conclude that in this case $\xi_{i}\left(v_{b}\right)=\xi_{j}\left(v_{b}\right)$ for any $i, j \in N, i \neq j$. Then the efficiency and symmetry axioms imply that $\xi\left(v_{b}\right)=\operatorname{Sh}\left(v_{b}\right)$.

Now suppose that $\xi\left(v_{b}\right)=\operatorname{Sh}\left(v_{b}\right)$ for all games $v_{b} \in \Im_{N}^{+}$with index not greater than some $q_{b} \geq 0$. Consider an arbitrary game $z_{b} \in \Im_{N}^{+}$with index $q_{b}+1$. For all $h, i \in \Theta=\bigcap_{k=1}^{q_{b}+1} T_{k}$ the symmetry axiom implies that $\xi_{h}\left(z_{b}\right)=\xi_{i}\left(z_{b}\right)$. Let $j \in N \backslash \Theta$ and $z_{b}^{\langle j\rangle} \in \Im_{N}^{+}$be the game defined by (9) for $x=b$. The index of $z_{b}^{\langle j\rangle}$ is at most $q_{b}$ and the $j$ th marginal utility vectors of the games $z_{b}^{\langle j\rangle}$ and $z_{b}$ coincide. Hence, by the marginalism axiom for $\xi$ and for the Shapley value and by induction hypothesis on $q_{b}$, for all $j \notin \Theta$ we have $\xi_{j}\left(z_{b}\right)=\xi_{j}\left(z_{b}^{\langle j\rangle}\right)=\operatorname{Sh}_{j}\left(z_{b}^{\langle j\rangle}\right)=\operatorname{Sh}_{j}\left(z_{b}\right)$. Next, by the efficiency axiom for $\xi$ as well as by the symmetry of the players belonging to the set $\Theta$ we conclude that $\xi_{i}\left(z_{b}\right)=\operatorname{Sh}_{i}\left(z_{b}\right)$ for any $i \in \Theta$. Thus, the proof is complete.

Of course, our remarks following the proof of Theorem 2 are valid also in this case. That is, the above procedure can be generalized to $\Im_{N}$ in a similar way.

3.3. Proof of Theorem 5. $(\Leftarrow)$ It is well-known (cf. J. Deegan and E. W. Packel (1978)) that the Deegan-Packel value satisfies the axioms given.

$(\Rightarrow)$ We know (J. Deegan and E. W. Packel (1978)) that each game $v \in G_{N}$ can be uniquely represented as a linear combination of basic games, 
that is,

$$
v=\sum_{\emptyset \neq T \subseteq N} \gamma_{T} w_{T}
$$

for some real constants $\gamma_{T}, T \subseteq N$.

The Deegan-Packel value of the game $w_{T}$ has the following form:

$$
\operatorname{DP}_{i}\left(w_{N}\right)=1 / n \text { and } \operatorname{DP}_{i}\left(w_{T}\right)= \begin{cases}1 /|T| & \text { if } i \in T, \\ 0 & \text { if } i \notin T,\end{cases}
$$

if $T \subseteq N$ and $T \neq \emptyset, N$, for any $i \in N$.

Let $\xi$ be a value on $G_{N}$ satisfying the quasi-efficiency, zero-player, symmetry and quasi-marginalism axioms. Assume that $q$ is the number of nonzero coefficients under the summation sign in (10). We will apply the induction procedure on $q$.

If $q=1$ then $v=\gamma_{T} w_{T}$ for some $T \subseteq N$. Thus, by the symmetry, quasi-efficiency and zero-player axioms for $\xi$ (note that any player belonging to $N \backslash T$ is a zero-player in the game $\left.w_{T}\right)$ we have $\xi(v)=\xi\left(\gamma_{T} w_{T}\right)=$ $\operatorname{DP}\left(\gamma_{T} w_{T}\right)=\gamma_{T} \cdot \operatorname{DP}\left(w_{T}\right)$.

Suppose that $\xi(v)=\operatorname{DP}(v)$ for all games $v \in G_{N}$ with index not greater than $q$. Let $z \in G_{N}$ be a game of the form (10) with $q+1$ nonzero coefficients in the summation sign. Thus

$$
z=\sum_{k=1}^{q+1} \gamma_{T_{k}} w_{T_{k}}
$$

where $\gamma_{T_{k}} \neq 0, k=1, \ldots, q+1$.

Put $\Theta=\bigcap_{k=1}^{q+1} T_{k}$. For all $h, i \in \Theta$ the symmetry of $\xi$ implies that $\xi_{h}(z)=\xi_{i}(z)$. We will prove that $\xi_{j}(z)=\mathrm{DP}_{j}(z)$ for any $j \in N \backslash \Theta$. Define a game $z^{\langle j\rangle} \in G_{N}$ such that

$$
z^{\langle j\rangle}=\sum_{k: j \in T_{k}} \gamma_{T_{k}} w_{T_{k}}
$$

The index of $z^{\langle j\rangle}$ is at most $q$ and therefore, by induction hypothesis, we have $\xi_{j}\left(z^{\langle j\rangle}\right)=\operatorname{DP}_{j}\left(z^{\langle j\rangle}\right)$. Since $j \notin \Theta$, player $j$ is a zero-player in all games $w_{T}$ with $j \notin T$. Consequently, for any $j \notin \Theta$ the $j$ th quasi-marginal utility vectors of the games $z$ and $z^{\langle j\rangle}$ coincide and hence, by the quasi-marginalism axiom for $\xi$, it follows that $\xi_{j}(z)=\xi_{j}\left(z^{\langle j\rangle}\right)=\mathrm{DP}_{j}\left(z^{\langle j\rangle}\right)=\mathrm{DP}_{j}(z), j \in N \backslash \Theta$. Hence, using the quasi-efficiency of $\xi$, we obtain

$$
\sum_{i \in \Theta} \xi_{i}(z)+\sum_{j \in N \backslash \Theta} \xi_{j}(z)=\sum_{S \subseteq N} z(S),
$$

and by symmetry of players belonging to $\Theta$ we conclude that $\xi_{i}(z)=\mathrm{DP}_{i}(z)$ for any $i \in \Theta$. 
REMARK. It is worth noting that in contrast to the previous theorems we have some difficulty in formulating a similar axiomatization of the DeeganPackel value defined on $\Gamma_{N}$ or $\Im_{N}$ (i.e., specific to those classes of games). First we must give up the zero-player axiom. This can be justified as follows. Let $v_{1} \in \Gamma_{N}, v_{2} \in \Im_{N}$ and $i \in N$ be a zero-player of both games. Then $0=v_{1}(S)=v_{1}(N)-v_{1}(N \backslash S)$ for any $S \ni i$. Hence, $v_{1}(N)=0$ and $v_{1}(A)=0$ for any $A \subseteq N$. Analogously, $0=v_{2}(S)=v_{2}(N \backslash S), v_{2}(N)=0$ and $v_{2}(A)=0$ for any $A \subseteq N$. Therefore for any nontrivial constant-sum or bilateral game no member of the grand coalition is a zero-player. Thus, the zero-player property is not useful here.

Consider a value $\xi$ defined on $\Im_{N}$ which is quasi-efficient, symmetric and quasi-marginalist. Let $v \in \Im_{N}$. Denote by $\phi_{i}(v), i \in N$, the $i$ th quasimarginal utility vector of $v$. If $N \ni j \neq i$ is another player of this game, then we obtain $\{v(S): S \subseteq N, i \in S\}=\{v(S): S \subseteq N, i, j \in S\} \cup\{v(S): S \subseteq N$, $i \in S, j \notin S\}=\{v(S): S \subseteq N, i, j \in S\} \cup\{v(N \backslash S): S \subseteq N, i \in S, j \notin S\}=$ $\{v(S): S \subseteq N, i, j \in S\} \cup\{v(S): S \subseteq N, i \notin S, j \in S\}=\{v(S): S \subseteq N$, $j \in S\}$ and hence $\phi_{i}(v)=\phi_{j}(v)$.

So, by quasi-marginalism and quasi-efficiency of $\xi$, we have

$$
\xi_{i}(v)=\frac{\sum_{S \subseteq N} v(S)}{n}=\mathrm{DP}_{i}(v)
$$

for any $i \in N$. Therefore, it is easy to observe that the requirement of symmetry of $\xi$ is redundant. The situation here is similar to the case of symmetric games, but a bilateral game need not be symmetric (a counterexample is e.g. the three-person game $v$ defined as $v(\{1\})=v(\{2\})=v(\{1,3\})=$ $v(\{2,3\})=0, v(\{3\})=v(\{1,2\})=1$ and $v(\{1,2,3\})=v(N)=2)$.

The case of $\Gamma_{N}$ seems to be slightly more sophisticated. There exists some premise suggesting that the construction of an effective axiomatization may not be possible by known methods. Let $\xi$ be a value defined on $\Gamma_{N}$ satisfying the quasi-efficiency, symmetry and quasi-marginalism axioms. Denote by $\omega_{T}$ a basis of $\Gamma_{N}$ indexed by $T \subseteq N$. That is, $\omega_{T} \in \Gamma_{N}, T \subseteq N$, are simple games such that any game $v \in \Gamma_{N}$ can be uniquely represented as

$$
v=\sum_{T \subseteq N} \chi_{T} \omega_{T}
$$

for some real constants $\chi_{T}, T \subseteq N$. Let $q$ be the number of nonzero coefficients in (11). Thus

$$
v=\sum_{k=1}^{q} \chi_{T_{k}} \omega_{T_{k}} .
$$

Let $\Theta=\bigcap_{k=1}^{q} T_{k}$ and $i \notin \Theta$. Putting $Q=\{1, \ldots, q\}$ we obtain 


$$
v=\sum_{k \in Q: i \in T_{k}} \chi_{T_{k}} \omega_{T_{k}}+\sum_{k \in Q: i \notin T_{k}} \chi_{T_{k}} \omega_{T_{k}} .
$$

In order that the $i$ th quasi-marginal utility vectors of the games $v$ and $\sum_{k \in Q: i \in T_{k}} \chi_{T_{k}} \omega_{T_{k}}$ coincide it is necessary and sufficient that $\vartheta(S):=$ $\sum_{k \in Q: i \notin T_{k}} \chi_{T_{k}} \omega_{T_{k}}=0$ whenever $S \ni i$. But $\vartheta \in \Gamma_{N}$ is a nontrivial constantsum game and $i$ must be a zero-player in $\vartheta$. As we noted earlier, this is impossible.

Acknowledgements. I am grateful to the anonymous referee for carefully reading the paper as well as for useful comments and suggestions.

\section{References}

J. F. Banzhaf III (1965), Weighted voting does not work: a mathematical analysis, Rutgers Law Rev. 19, 317-343.

J. Deegan and E. W. Packel (1978), A new index of power for simple n-person games, Internat. J. Game Theory 7, 113-123.

A. B. Khmelnitskaya (2003), Shapley value for constant-sum games, ibid. 32, 223-227.

E. Lehrer (1988), An axiomatization of the Banzhaf value, ibid. 17, 89-99.

S. C. Littlechild and G. Owen (1976), A further note on the nucleolus of the "airport game", ibid. 5, 91-95.

A. Młodak (2003), Three additive solutions of cooperative games with a priori unions, Appl. Math. (Warsaw) 30, 69-87.

G. Owen (1977), Values of games with a priori unions, in: Mathematical Economics and Game Theory, R. Henn and O. Moeschlin (eds.), Springer, Berlin, 76-88.

J. A. M. Potters (1991), An axiomatization of the nucleolus, Internat. J. Game Theory $19,365-373$.

L. M. Ruiz, F. Valenciano and J. M. Zarzuelo (1996), The least square prenucleolus and the least square nucleolus. Two values for TU games based on the excess vector, Internat. J. Game Theory 25, 113-134.

L. S. Shapley (1953), A value for n-person game, in: Ann. of Math. Stud. 28, Princeton Univ. Press, Princeton, NJ, 307-317.

M. Vázquez-Brage, A. Van den Nouweland and I. García-Jurado (1997), Owen's coalitional value and aircraft landing fees, Math. Social Sci. 34, 273-286.

H. P. Young (1985), Monotonic solutions of cooperative games, Internat. J. Game Theory $14,65-72$.

Central Statistical Office

Statistical Office in Poznań

Branch in Kalisz

Pl. J. Kilińskiego 13

62-800 Kalisz, Poland

E-mail: a.mlodak@stat.gov.pl

Received on 4.11.2004;

revised version on 24.8.2007 
